\title{
RESÍDUOS POTENCIALMENTE INFECTANTES EM SERVIÇOS DE HEMOTERAPIA E AS INTERFACES COM AS DOENÇAS INFECCIOSAS
}

\author{
Marinésia Aparecida do Prado* \\ Dulcelene de Sousa Melo** \\ Katiusse Macedo Machado*** \\ Silvana de Lima Vieira dos Santos** \\ Elucir Gir**** \\ Silvia Rita Marin da Silva Canini ${ }^{\star \star \star \star \star ~}$ \\ Nilza Tereza Rotter Pelá ${ }^{\star \star * * * \star}$
}

\section{Resumo}

Objetivou-se identificar o processamento de resíduos potencialmente infectantes e de perfurocortantes em unidades de hemoterapia e sua possível relação com a saúde dos trabalhadores. Nenhum serviço apresentou plano estruturado para o gerenciamento de resíduos de serviços de saúde e para o programa de segurança ocupacional. Verificouse um índice de acidentes ocupacionais de 32 , $82 \%$ e que $9,16 \%$ dos trabalhadores estavam com esquema incompleto de vacinação contra hepatite B. Os treinamentos em serviços para manejo de resíduos e saúde ocupacional não são padronizados. O índice de soroconversão para VHB e VHC após exposição ocupacional foi de $11,63 \%$. Concluímos que o processamento de resíduos potencialmente infectantes e perfurocortantes nas unidades de hemoterapias pesquisadas tem exposto os trabalhadores à aquisição de doenças infecciosas.

Descritores: bancos de sangue; resíduos de serviços de saúde; acidentes de trabalho

\begin{abstract}
The aim was to identify the processing of potentially infectious residues and of piercing and cutting residues at hemotherapy units and their possible relation with workers' health. No service presented a structured plan for managing health service residues or for the occupational safety program. We found an occupational accident rate of $32.82 \%$ and that $9.16 \%$ of the workers did not have a complete plan of vaccination against hepatitis $B$. Inservice training in handling residues and in occupational health are not standardized. The rate of serum conversion for HBV and $\mathrm{HCV}$ after occupational exposure was $11.63 \%$. We concluded that the processing of potentially infectious residues and of piercing and cutting residues at the examined hemotherapy units has exposed workers to the acquisition of infectious diseases.

Descriptors: blood banks; health service residues (waste); occupational accidents Title: Potentially infectious residues at hemotherapy services and interfaces with infectious diseases
\end{abstract}

\section{Resumen}

La finalidad fue la de identificar el procesamiento de residuos potencialmente infectivos, cortantes y punzantes en unidades de hemoterapia y su posible relación con la salud de los trabajadores. Ningún servicio presentó plan estructurado para la gestión de residuos de servicios de salud y para el programa de seguridad ocupacional. Se observó un índice de accidentes ocupacionales de $32,82 \%$ y que $9,16 \%$ de los trabajadores estaban con plan incompleto de vacunación contra hepatitis B. La capacitación en servicios para manejo de residuos y salud ocupacional no está estandardizada. El índice de seroconversión para VHB y VHC tras exposición ocupacional fue de 11,63\%. Concluimos que el procesamiento de residuos potencialmente infectivos, cortantes $y$ punzantes en las unidades de hemoterapia investigadas ha expuesto a los trabajadores a la adquisición de enfermedades infecciosas.

Descriptores: bancos de sangre; residuos de hospitales; accidentes de trabajo

Título: Residuos potencialmente infectivos en servicios de hemoterapia y las interfaces con las enfermedades infecciosas

\section{Introdução}

Atualmente estamos inseridos num contexto em que, ora beneficia, ora promove a inadequação das condições que assegurem à manutenção da vida. Tal situação contribui para a cadeia epidemiológica das doenças infecciosas.

A tecnologia em seus avanços de alta complexidade tem possibilitado o dinamismo na evolução e na produção do conhecimento nas várias atividades humanas.

Em contrapartida, tem possibilitado mudanças no comportamento e nas necessidades humanas, o que tem gerado problemas éticos e ambientais e, que comprometem o equilíbrio da ecologia em seu sentido pleno(1). $^{(1)}$

Entretanto, há de considerar o processo de transformação ocorrido no âmbito cultural, social e econômico vivenciado no momento, onde os impactos relacionados à tecnologia e ao comportamento da sociedade têm refletido na manutenção do processo saúde-doença das pessoas. Assim, entre outros fatores, associados à prática do cotidiano das instituições de saúde geradoras de resíduos potencialmente infectantes e perfurocortantes, como por exemplo, os serviços de hemoterapia, deve ser repensada, para que as conseqüências negativas destas ações não inviabilizem a vida ${ }^{(2)}$.

É importante ressaltar que, o modelo de organização da sociedade moderna dificulta o entendimento por parte do indivíduo quanto a sua participação, enquanto, controlador dos fatores de risco e dos problemas ambientais, em que, especialmente o manejo adequado, destes resíduos seja assegurado(2).

Os Resíduos de Serviços de Saúde (RSS), constituem uma categoria específica dos resíduos sólidos devido a suas particularidades, especialmente em razão da presença de resíduos que oferecem riscos biológicos. Portanto, estes resíduos resultam de atividades da comunidade de origem industrial, doméstica, hospitalar, comercial, agrícola, de serviços e de varrição(2-4)

\footnotetext{
* Enfermeira. Professor Assistente da Faculdade de Enfermagem da Universidade Federal de Goiás. Doutoranda em enfermagem do Programa de Interunidades da USP. **Enfermeira com Especialização em enfermagem em infectologia pela EERP/USP. Mestranda em enfermagem da FEN/UFG. ***Enfermeira do Instituto Goiano de Hemoterapia de Anápolis/Goiás. Especialização em enfermagem em infectologia pela EERP/USP. **Enfermeira com Especialização em enfermagem em infectologia pela EERP/USP. Mestranda em enfermagem da FEN/UFG. ****Enfermeira. Professora Associada

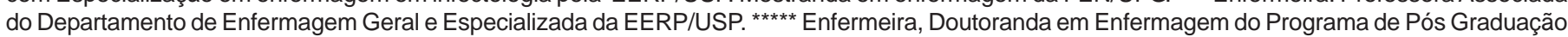
de Enfermagem Fundamental da Escola de Enfermagem de Ribeirão Preto da Universidade de São Paulo. ${ }^{\star \star \star \star \star \star}$ Enfermeira. Professor Titular do Departamento de Enfermagem Geral e Especializada da EERP/USP.

E-mail do autor: prado@aol.com
} 
Ressaltamos que, segundo a regulamentação da Agência Nacional de Vigilância Sanitária (ANVISA) RDC 33 de 25/02/03, são geradores de RSS, todos os serviços que prestam atendimento à saúde humana ou animal, incluindo os programas de assistência domiciliar, indústrias e serviços de pesquisa na área de saúde, hospitais, clínicas, ambulatórios médico e odontológicos, acupuntura, tatuagem, drogarias, farmácias, tratamento quimioterápico, radioterapia e de medicina nuclear, unidades de produção de hemoderivados, laboratórios de análises clínicas e patológica, necrotérios e medicina legal, unidades de controle de zoonozes e unidades móveis de atendimento à saúde, entre outros ${ }^{(5)}$.

Portanto o gerenciamento de RSS constitui-se, em um conjunto de procedimentos de gestão, planejados e implementados a partir de bases científicas e técnicas normativas e legais, com o objetivo de minimizar a produção de resíduos, de forma eficiente, visando a proteção dos trabalhadores, a preservação da saúde pública e dos recursos naturais e do meio ambiente ${ }^{(2,5)}$.

De acordo com a portaria $n^{0} 3.214 / 78$ - NR - 09 do Ministério do Trabalho, constitui-se em resíduo com risco biológico, aqueles com presença de microrganismos (bactérias, fungos, bacilos, parasitas, protozoários, vírus entre outros) capazes de causar infecção no homem e disseminar doenças ${ }^{(6)}$

Nesse contexto a classificação destes resíduos, preconizada pelas resoluções 05/93 e 283/01 do Conselho Nacional do Meio Ambiente (CONAMA), tendo como base à composição e as características biológicas, encontram-se distribuídas em quatro grupos, a saber: $A, B, C$ e $D^{(3)}$

Entretanto com a nova regulamentação técnica da Agência Nacional de Vigilância Sanitária, estes resíduos foram reclassificados em cinco grupos: $A, B, C, D$ e E, em conformidade com esta regulamentação, as Instituições de Assistência à Saúde, foram convocadas a elaborarem ou reformularem o seu plano de gerenciamento de RSS, adequando-o às mesmas, em um prazo máximo de 12 meses, a partir da data de sua publicação(5).

Os resíduos gerados nos serviços de hemoterapia de acordo com a legislação vigente enquadraram-se nos seguintes grupos:

Grupo A: bolsas de sangue ou hemocomponentes com volume residual superior a $50 \mathrm{ml}$ e kits de aférese.

Grupo D: bolsas transfundidas vazias ou contendo menos de $50 \mathrm{ml}$ de produto residual (sangue ou hemocomponentes); invólucros de materiais como: papéis, plásticos, etc.

Grupo E: bolsas de coleta incompleta descartada no local da coleta quando acompanhadas de agulhas, independente do volume coletado e agulhas hipodérmicas.

Assim os serviços de hemoterapia devem elaborar um plano de gerenciamento de RSS, dentro da atual classificação, uma vez que, os resíduos ali gerados são considerados resíduos biológicos, em particular os potencialmente infectantes e os perfurocortantes, tendo como premissa a carga residual presente neste material e os riscos que tais materiais representam para as pessoas, bem como para o meio ambiente.

Diante destas informações e da nossa vivência em algumas instituições de saúde e como pesquisadoras atuando há alguns anos na prevenção e no controle das infecções hospitalares e medidas de biossegurança em estabelecimentos de saúde, percebemos que o gerenciamento dos RSS ainda é ineficaz, o que favorece a cadeia epidemiológica das doenças infecciosas e a exposição de trabalhadores aos acidentes envolvendo materiais biológicos.

Acreditamos que este ciclo compromete a qualidade dos serviços de saúde oferecidos à população, o que justifica a realização de estudos que busquem compreender 0 gerenciamento dos resíduos potencialmente infectantes e perfurocortantes em serviços de hemoterapia das instituições de saúde e suas interfaces com as doenças infecciosas.

Neste contexto elaboramos os seguintes questionamentos:
Como o gerenciamento dos resíduos potencialmente infectantes e perfurocortantes nos serviços de hemoterapia está sendo operacionalizado?

O gerenciamento dos resíduos potencialmente infectantes e perfurocortantes, uma vez, inadequado tem exposto as pessoas ao risco de adquirir doenças infecciosas intra e extra instituição geradora?

Quais os fatores que facilitam ou que dificultam o gerenciamento adequado destes resíduos?

Há protocolo norteando a prática do gerenciamento de resíduos do serviço de saúde nas instituições?

Nessa ótica partimos do pressuposto de que, o gerenciamento dos resíduos infectantes, uma vez realizados, inadequadamente, nos serviços de hemoterapia expõe os indivíduos ao risco de adquirir doenças infecciosas tanto intra como extra instituição geradora. O protocolo para o manejo dos resíduos potencialmente infectantes e dos perfurocortantes constitui uma ferramenta essencial para o gerenciamento seguro dos mesmos, sendo considerado um agente facilitador para os serviços que já o possuem.

\section{Objetivos}

Verificar se o processamento dos resíduos potencialmente infectantes e os perfurocortantes favorecem a exposição dos indivíduos ao risco de adquirirem doenças infecciosas intra e extra instituição de saúde geradora;

Identificar os fatores facilitadores e ou dificultadores relacionados ao gerenciamento adequado destes resíduos; Identificar e avaliar os indicadores utilizados acerca da normatização e do manejo dos resíduos infectantes e os perfurocortantes produzidos nos serviços de hemoterapia em instituições de saúde do município de Goiânia - GO.

\section{Material e Método}

Pesquisa do tipo survey, a qual permite a obtenção de informações por meio de auto-relatos, foi realizada no período de maio a novembro de 2003 em Goiânia-GO. Participaram 6 serviços de hemoterapia, sendo 2 públicos, 2 privados e 2 filantrópicos, os quais foram classificados como unidades 1, 2, 3, 4, 5 e 6 .

A população foi constituída por profissionais e por responsáveis pelo gerenciamento, manuseio e processamento interno dos resíduos potencialmente infectantes e perfurocortantes lotados nos serviços de hemoterapia. Foram excluídos os profissionais que se encontravam afastados temporariamente de suas atividades por licenças: maternidade, prêmio, saúde ou de interesse particular e não se encontravam no momento da coleta ou ainda que se recusaram a integrar formalmente à pesquisa.

Participaram da pesquisa 203 profissionais de seis serviços de hemoterapia sendo 02 públicos, 02 privados e 02 filantrópicos, os quais foram identificados como unidades 1, 2 , $3,4,5$ e 6 . O número de profissionais por serviço variou de 14 a 98, totalizando 203 profissionais. A amostra do estudo foi composta por 131 sujeitos da pesquisa, os quais estavam em atividade no período da coleta e concordaram em participar.

Este projeto foi aprovado pelos Comitês de Ética em Pesquisa da Universidade Federal de Goiás e Santa Casa de Misericórdia de Goiânia. As informações foram coletadas após a exposição dos objetivos da pesquisa aos sujeitos e a sua concordância expressa por meio do termo de livre esclarecido.

Em seguida aplicou-se um questionário contendo questões abertas e fechadas aos sujeitos. A coleta de dados foi complementada por meio de observação direta, não participativa, em um único momento, quando as pesquisadoras assinalavam um roteiro de observação simples (check-list) previamente validados, por especialistas na temática. Por último foi realizado um teste piloto em uma instituição de hemoterapia de outro município, o qual não foi incluído neste estudo. O banco de dados foi estruturado no programa Excel 2000 e os resultados analisados por meio de estatística descritiva. 


\section{Resultados e discussão}

Verificou-se que os serviços pesquisados geram resíduos dos grupos $A, B, D$ e $E$, conforme classificação da legislação vigente ${ }^{(5)}$. Os serviços 5 e 6 produzem os resíduos do grupo $B$ em maior escala que os outros, uma vez que realizam atividades envolvendo administração de quimioterápicos. Há de se considerar que, cada grupo de resíduos, exige uma demanda diferenciada para seu manejo, no entanto, neste estudo o enfoque foi dado aos resíduos dos grupos A e E.

Quanto a distribuição dos sujeitos por categoria funcional houve maior incidência de técnicos de laboratório 20,61\%, seguidos por $19,08 \%$ biomédicos e $19,08 \%$ auxiliares de serviço geral e $15,27 \%$ técnicos em enfermagem.

Com relação ao tempo de atuação profissional em serviços de hemoterapia, verificou-se que, estes profissionais, estão exercendo suas atividades entre 1 e 5 anos (39,69\%).

O plano de gerenciamento de resíduos dos serviços de saúde (PGRSS), documento que descreve as ações relativas ao manejo dos RSS deve ser compreendido em todas as suas fases inclusive quanto a proteção à saúde pública, considerado o principal indicador de qualidade e de segurança à saúde do trabalhador, da comunidade e minimizador de agravos ao meio ambiente ${ }^{(5)}$.

Nesse sentido, considerou-se pertinente indagar os profissionais acerca do manejo de resíduos com risco biológico, implementado nos serviços. Identificou-se que quatro serviços possuem planos de gerenciamento e dois não. Em dois serviços o mesmo foi elaborado a partir da publicação da nova legislação, porém nenhum ainda está implementado.

O Ministério da Saúde do Brasil, disponibilizou curso gratuito de capacitação à distância, direcionado aos profissionais responsáveis pelo gerenciamento dos RSS de várias Instituições de Saúde do país, com o objetivo de minimizar o impacto da prática do manejo inadequado destes resíduos até então adotados(2).

Nesse enfoque investigou-se junto aos profissionais dos serviços de hemoterapia sobre o Plano de Gerenciamento PGRSS adotado na instituição e verificou-se que, apesar dos profissionais saberem da existência do mesmo, este ainda se encontra em fase de elaboração em 4 serviços e em fase inicial de implementação em 2 serviços, entretanto nenhum serviço os validou. Podemos concluir que nenhum serviço pesquisado possui PGRSS estruturado, implantado e já avaliado.

Com relação à normatização para o exercício das atividades do manuseio interno dos RSS, detectou-se que 5 serviços a disponibilizam, estando documentada e inserida em sua rotina e 1 não a disponibilizou.

As normatizações devem ser padronizadas e detalhadas de forma clara, proporcionando o seu entendimento por todos os profissionais envolvidos, promovendo assim, as adequações necessárias ${ }^{(7)}$.

No tocante às fases que compreendem o manejo interno dos resíduos potencialmente infectantes e perfurocortantes, foi observado que, a segregação e o acondicionamento, realizados nos serviços de hemoterapia, não atendem as orientações estabelecidas, uma vez que, a separação e o ato de embalar não atendem todos os critérios definidos pela resolução do Ministério da Saúde ${ }^{(1,2,5)}$.

Ressalta-se que em 5 serviços observados, os resíduos pertencentes aos grupos A e D são segregados seguindo as orientações daqueles classificados no grupo $A$, o que é contra indicado, pois, segundo a recomendação vigente os resíduos do grupo D, devem ser segregados em sacos plásticos comuns, o que torna o seu custo mais acessível para o serviço.

Para os resíduos do grupo B percebe-se a adoção de condutas diferenciadas e que também não atendem a legislação vigente, percebe-se que esta deficiência tem seu início desde a sua estruturação em nível de órgãos fiscalizadores de forma a esclarecer e estabelecer tais condutas.

Quanto ao serviço de atendimento ao trabalhador exposto a acidente ocupacional, todos os serviços investigados o disponibilizam. No que se refere a normatização do fluxo de atendimento em caso de acidentes entre os profissionais observou-se que apenas um dos serviços não a possui. Entretanto, todos os serviços efetivam o registro e o acompanhamento dos profissionais vítimas de acidentes ocupacionais, cuja atribuição em 4 serviços fica a cargo do Serviço de Controle de Infecção Hospitalar ou da Comissão de Controle de Infecção Hospitalar - SClH/CCIH.

Vale ressaltar que o Serviço de Controle de Infecção tem como objetivos: o planejamento, a implementação e a avaliação de medidas direcionadas ao controle de ações educativas que promovam a mudança de comportamento, acerca do controle de infecção, partindo do individual para o coletivo; colaborar com a CCIH na monitorização e investigação de exposições a agentes potencialmente infecciosos e em surtos entre os profissionais; prover assistência ao pessoal em casos de exposição ou doenças relacionadas ao trabalho; identificar riscos de infecção relacionados ao trabalho e as medidas apropriadas e conter custos prevenindo doenças infecciosas que resultem em absenteísmo e incapacidade na ausência de um serviço de Medicina do Trabalho(8).

As atividades desenvolvidas pelos profissionais nos serviços de hemoterapia tornam-os susceptíveis a riscos de acidentarem com maior freqüência, nesse sentido, preocupamo-nos em identificar a ocorrência de acidentes ocupacionais. Verificou-se que dos $32,82 \%$ trabalhadores acidentados, $41,86 \%$ foram expostos mais de uma vez.

O material biológico envolvido com maior freqüência foi o sangue, referido por $73,33 \%$ dos profissionais. O sangue é o material manipulado com maior freqüência pelos profissionais dos serviços de hemoterapia, tornando-os mais expostos aos riscos biológicos, visto que parte da demanda espontânea destes serviços, emerge para a verificação do estado sorológico da população em situação de risco e de estarem contaminados com agentes infecciosos de importância epidemiológica.

Quanto ao tipo de exposição detectou-se que, 39,13\% foram vítimas de acidentes com materiais perfurantes e $23,92 \%$ de perfurocortantes.

Vale ressaltar que estes materiais representam uma fonte de contaminação de maior relevância na prática dos trabalhados de saúde, uma vez que, contém material biológico e são capazes de veicular microrganismo como os vírus das hepatites $\mathrm{B}$ (VHB) e C (VHC) e do Vírus da Imunodeficiência Humana (HIV) ${ }^{(1,7,9)}$. Estes resultados encontram-se evidenciados no Gráfico 1.

$\mathrm{Em}$ relação às condutas tomadas após o acidente $21,05 \%$ profissionais realizaram esquema completo dos exames de sorologias e 10,53\% sorologias do cliente. A limpeza do local com água e sabão e a utilização de anti-séptico embora seja de grande importância, foi relatado por apenas 1,05\% dos profissionais e 1,05\% não realizou nenhuma conduta, sendo esta considerada falha grave do sistema de atendimento no tocante a avaliação dos agravos eminentes da saúde do trabalhador.

A tabela 1 apresenta a distribuição das condutas adotadas pelos profissionais após acidentarem.

Apesar dos profissionais saberem da necessidade de atendimento em caso de acidente ocupacional não observamos haver uniformidade quanto às condutas adotadas por eles após o acidente. Os profissionais não têm clareza sobre a necessidade do seguimento do protocolo de controle pós-exposição ocupacional à material biológico. Segundo as recomendações preconizadas estas devem ser elaboradas, implementadas e divulgadas à equipe profissional com o intuito de padronizar os procedimentos a serem seguidos na ocorrência do mesmo, visando à prevenção, tratamento e acompanhamento(10). 


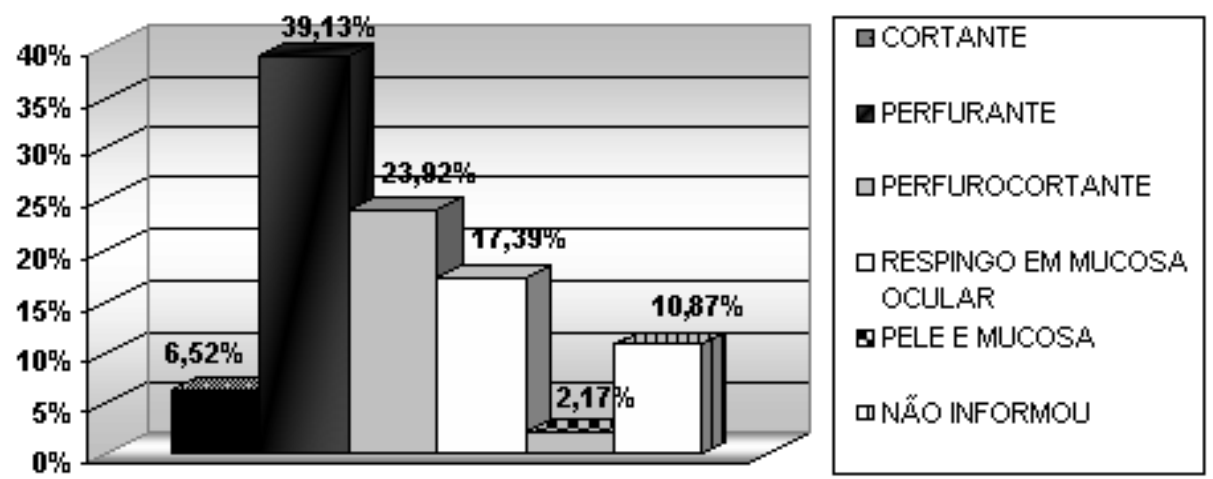

Gráfico 1- Distribuição dos tipos de acidentes ocorridos entre os profissionais dos serviços de hemoterapia. Goiânia/2003.

Tabela 1 - Distribuição das condutas adotadas após o acidente segundo relatos dos profissionais dos serviços de hemoterapia. Goiânia (GO), 2003.

\begin{tabular}{lrr}
\hline \multicolumn{1}{c}{ Conduta frente ao acidente } & Freqüência & $\%$ \\
\hline Limpeza do local com água e sabão & 7 & 7,37 \\
Limpeza com água + sabão + álcool 70\% & 1 & 1,05 \\
Limpeza com hipoclorito.+ água + sabão & 5 & 5,26 \\
Consulta médica & 2 & 2,11 \\
Encaminhadas ao Hospital Doenças Tropicais & 8 & 8,42 \\
Acompanhamento por um ano & 3 & 3,16 \\
Acompanhamento por 6 meses & 6 & 6,32 \\
Sorologia do cliente & 10 & 10,53 \\
Sorologias completas & 20 & 21,05 \\
Sorologia após 1 mês & 2 & 2,11 \\
Imunoprofilaxia & 6 & 6,32 \\
Notificou a CCIH & 11 & 11,58 \\
Comunicou à chefia imediata & 2 & 2,11 \\
Outros & 10 & 10,53 \\
Não informou & 2 & 2,11 \\
\hline \multicolumn{1}{c}{ TOTAL } & & 100 \\
\hline
\end{tabular}

Ao se acidentar o profissional deve adotar as seguintes condutas ${ }^{(10-13)}$ :

- Lavar o local lesionado abundantemente com água e sabão, seguido da aplicação de solução anti-séptica (álcool a 70\%, PVPI ou Clorexidina) e, em caso de envolvimento de mucosa, utilizar soro fisiológico 0,9\% ou água boricada, e na ausência destes, água corrente ${ }^{(10)}$.

- Notificar imediatamente à chefia, a qual notifica a CCIH ou setor que avaliará o acidente, o mais precoce possível, idealmente até duas horas após o acidente, pois, a eficácia das medidas profilática, pós-exposição é maior quando oferecida o mais precocemente possível, sobretudo quando 0 acidente caracterizar-se como grave e o paciente fonte for soro reagente para HIV.

- Realizar a comunicação do acidente de trabalho (CAT).

- Coletar sangue do paciente fonte e do profissional acidentado para sorologias.

- Analisar os dados da fonte de contágio, causas do acidente, tipo de acidente.

- Adotar medidas pertinentes a vacinas, quimioprofilaxia e outras.

- Realizar as orientações aplicáveis no plano individual e familiar.

- Seguimento clínico-laboratorial apropriado de 6 meses a 18 meses ${ }^{(10-13)}$.

Quanto à utilização de equipamentos de proteção individual (EPI), 99,24\% dos profissionais referem que os utilizam. Entretanto em algumas situações o utiliza de maneira inadequada. Ressalta-se que o uso do EPI está recomendado a todo profissional no atendimento a todo cliente independente de seu presumível estado de saúde e quando houver possibilidade de exposição a sangue, líquidos e secreções corpóreas ${ }^{(14) .}$ Apesar dessa recomendação, o uso das luvas não foi mencionado por 3,27\% dos profissionais, fator relevante considerando-se a especificidade de suas atividades.

Os EPI utilizados com maior freqüência, segundo os relatos dos profissionais participantes foram luvas $27.02 \%$, jalecos $19,78 \%$, óculos $17,65 \%$ e máscaras $16,80 \%$. O protetor facial embora importante para o tipo de atividade desenvolvida pelos profissionais nos serviços de hemoterapia foi referido por apenas 2,34\% deles. Estes resultados encontram-se disponibilizados na Tabela 2.

Tabela 2 - Distribuição dos EPI utilizados pelos profissionais nos serviços de hemoterapia. Goiânia-GO. 2003.

\begin{tabular}{lcc}
\hline Equipamentos de Proteção Individual & Freqüência & $\%$ \\
\hline Luvas & 127 & 27,02 \\
Máscara & 79 & 16,80 \\
Óculos & 83 & 17,65 \\
Jaleco & 93 & 19,78 \\
Avental/Capote & 8 & 1,71 \\
Protetor facial & 11 & 2,34 \\
Botas & 20 & 4,25 \\
Gorro & 15 & 3,20 \\
Sapato fechado & 18 & 3,82 \\
Avental plástico & 1 & 0,22 \\
Capacete & 1 & 0,22 \\
Uniforme privativo & 6 & 1,28 \\
Outros & 6 & 1,28 \\
Não informou & 2 & 0,43 \\
\hline
\end{tabular}

Vale ressaltar que segundo as recomendações da NR6 do Ministério do Trabalho, os EPI de uso cotidiano dos serviços de hemoterapia compreendem luvas, jalecos, máscaras , protetor facial e no caso específico do capote impermeável ele é recomendado em algumas situações.

Ao questionar os profissionais dos serviços de hemoterapia quanto ao treinamento sobre as normas de biossegurança e saúde ocupacional, 77,10\% afirmaram ter recebido treinamento e $22,14 \%$ relataram que nunca receberam nenhum tipo de treinamento. No tocante às orientações atinentes ao manejo de resíduos de saúde $80,92 \%$ profissionais referiram ter recebido orientações específicas e $19,08 \%$ não as receberam.

A periodicidade acerca das orientações relatadas pelos profissionais variou de diária até a cada dez anos. Pode-se inferir que estes serviços não têm priorizado as atividades de educação permanente relacionadas aos RSS ou que as mesmas não se encontram estabelecidas em cronogramas ou ainda não têm contemplado a participação de maneira uniforme por parte dos trabalhadores. 
Em qualquer atividade de prestação de serviços é recomendável que os profissionais de saúde sejam periodicamente atualizados quanto às normas e rotinas do serviço, bem como das medidas de biossegurança. As condições com potencial de causar danos aos trabalhadores são variadas e, para controlá-las é necessária a sistematização das atividades de educação continuada/permanente e assim auxiliar na promoção de mudança de comportamento destes profissionais.

Esse direito está assegurado nas novas normatizações da ANVISA ${ }^{(5)}$, cabendo aos dirigentes dos estabelecimentos geradores de RSS a responsabilidade quanto à capacitação, treinamento e manutenção de programas de educação permanente, direcionados ao pessoal envolvido no gerenciamento e manejo dos resíduos.

Compreendendo também o PGRSS como meio de desenvolvimento e de implantação de programas de capacitação que envolva os setores geradores destes resíduos, bem como, os serviços de higiene e limpeza, SCIH/CCIH, SESMT e CIPA. Esta capacitação deve ocorrer a partir da admissão dos trabalhadores nas empresas e deve ser mantido por meio de acompanhamento sistemático destes profissionais em suas atividades durante o manejo dos resíduos, inclusive aos profissionais em situação temporária ou que não estejam envolvidos diretamente nas atividades de manuseio dos mesmos ${ }^{(5)}$

A equipe de saúde deve ser educada sobre a política e procedimentos de controle de infecção da organização. Conseqüentemente isto possibilitará o desenvolvimento seguro das atividades de prestação de serviços de saúde do trabalhador e do cliente ${ }^{(8)}$.

Em relação ao esquema vacinal dos profissionais dos serviços pesquisados verificamos que estes o valorizam como fator de proteção para a atividade profissional, uma vez que, $79,38 \%$ referiram esquema completo para hepatite B. Porém, questionamos o fato de que $9,16 \%$ referiram esquema incompleto e 2,30\% não receberam nenhuma dose da vacina contra hepatite B. As vacinas constituem parte essencial dos programas bem sucedidos de saúde ocupacional, pois asseguram imunidade ao pessoal quanto às doenças preveníveis ${ }^{(8,11,15)}$.

No que se refere à triagem sorológica pós-vacinal dos profissionais dos serviços de hemoterapia de Goiânia, verificamos que $23,67 \%$ não realizaram. Constatamos por meio dos dados que há dúvidas por parte dos profissionais quanto à interpretação dos resultados das sorologias realizadas.

Esta conduta é recomendada aos trabalhadores que se encontram em situação permanente de exposição ao sangue, para verificar a dosagem de títulos cujo objetivo é conferir se o trabalhador adquiriu imunidade e auxiliar na profilaxia pósexposição ocupacional com indicação da revacinação ou mesmo indicação de gamaglobulina hiperimune específica ${ }^{(8,11,15)}$.

Dados registrados na literatura alertam para o risco de infecção ocupacional cujos agentes são veiculados pelo sangue e outros fluídos corpóreos, apontam ainda a maior infectividade do VHB em relação ao HIV, entre os profissionais da área da saúde e atingindo um percentual de suscetibilidade maior em relação à população em geral ${ }^{(1,16,17)}$.

Estes resultados encontram-se condizentes com os alcançados na presente pesquisa, uma vez que, 32,82\% dos profissionais dos serviços de hemoterapia relataram exposição ocupacional, destes $11,63 \%$ apresentaram soroconversão para VHB e VHC. Observa-se que alguns fatores como a duração e a freqüência com o sangue e derivados, bem como a positividade dos clientes para anti-HBsAg, são determinantes na infecção ocupacional( ${ }^{17)}$

\section{Conclusão}

Os objetivos foram contemplados e possibilitam pontuar alguns aspectos:

O tempo de atuação dos profissionais variou de 1 a 25 anos. Não existe plano de gerenciamento de resíduos de serviço de saúde estruturado nos serviços de hemoterapia pesquisados.

Os fatores dificultadores para o manejo adequado dos RSS foram, a falta de medidas administrativas e de engenharia; falta de um programa de segurança ocupacional estruturado, capaz de dar suporte de forma efetiva ao profissional em situação de acidente com material biológico.

- A adesão aos EPI recomendados foi mencionada por 99,24\% dos profissionais, entre eles destacaram-se as luvas, os óculos, as máscaras e os jalecos.

O índice de acidente foi de 32,82 \% entre os trabalhadores, sendo 55,82\% expostos uma vez e 41,86\% mais de uma vez.

- O material biológico envolvido com maior freqüência foi o sangue, em 73, 33\% dos acidentes ocupacionais.

O esquema completo para vacina contra hepatite $B$ foi referido por $79,38 \%$, porém, $9,16 \%$ referiram esquema incompleto e 2,30\% não receberam nenhuma dose.

- Quanto a sorologia (Anti-HbsAg) pós vacinal, 70,23\% dos profissionais a realizaram.

$77,10 \%$ dos profissionais referiram ter recebido treinamento quanto às medidas de biossegurança e saúde ocupacional ao ingressar no serviço.

- Q Quanto ao manejo dos resíduos de serviço de saúde, 80,92\% relataram ter recebido orientação.

$11,63 \%$ dos profissionais que referiram acidente ocupacional apresentaram soroconversão para VHB e VHC.

Frente aos resultados obtidos, consideramos que 0 gerenciamento de resíduos potencialmente infectantes e perfurocortantes em unidades de hemoterapias tem exposto os profissionais ao risco de adquirir doenças infecciosas.

\section{Considerações finais}

Embora nos pareça que os resultados da pesquisa sejam elucidativos, é importante alertar sobre a questão do gerenciamento dos resíduos de serviços de saúde, considerando o impacto que os mesmos representam à saúde da população, por um lado pelos possíveis agravos até o momento não mensurados e por outro pelas especificidades das atividades desenvolvidas pelos prestadores de serviços relacionados às unidades de hemoterapia, deixando de lado algumas temáticas e discussões para a prática.

Todos as unidades geradoras de RSS têm sua responsabilidade frente ao problema de saúde pública, uma vez que, o este tem reflexo direto na ecologia. Portando é necessário que nos atentemos para esta e outras problemáticas passíveis de intervenção.

Sob essa ótica, esta pesquisa traz contribuições de grande impacto para o saneamento ambiental e para as doenças infecciosas relacionadas tanto ao gerenciamento inadequado de resíduos potencialmente infectantes como aos materiais perfurocortantes em unidades de hemoterapias e o seu reflexo nas ciências da saúde, de significado epidemiológico indiscutível na população intra e extra instituição geradora.

Consideramos relevantes e prementes a estruturação do PGRSS, nestes serviços, bem como a elaboração de um cronograma direcionado as atividades de educação permanente em serviço e a elaboração de um protocolo que auxiliem a compreensão de todos os profissionais responsáveis pelo manuseio e gerenciamento dos resíduos potencialmente infectantes e perfurocortantes nos serviços de hemoterapias, com vistas a minimizar seus agravos e assegurar a saúde e a qualidade de vida dos cidadãos.

\section{Referências}

1. Ministério da Saúde(BR). Gerenciamento de resíduos de serviços de saúde. Projeto Reforço à Reorganização do Sistema Único de Saúde. Brasília (DF);2001.120p.

2. Ministério da Saúde(BR). Saúde Ambiental e Gestão de Resíduos de 
Serviços de Saúde. Brasília (DF); 2002.317p.

3. Conselho Nacional do Meio Ambiente (BR). Resolução $n^{0} 5$, de 05 de agosto de 1993. Define os procedimentos mínimos para o gerenciamento de resíduos sólidos provenientes de serviços de saúde, portos e aeroportos. Estende exigências aos terminais rodoviários e ferroviários. Disponível em: http://www.mma.gov.br/ port/conama/res/res93/res0593.html. Acessado em: 26 maio 2004.

4. Conselho Nacional do Meio Ambiente (BR).Resolução $n^{0} 283$, de 12 de julho de 2001. Dispõe sobre o tratamento e destinação final dos resíduos de serviços de saúde. Disponível em: http://www.mma. gov.br/port/conama/processos/98E7BD03/PropostaCetesbAblp Preto.doc>. Acessado em : 26 maio 2004.

5. Ministério da Saúde (BR). Agencia Nacional de Vigilância Sanitária. Resolução de Diretoria Colegiada $n^{0} n$ 33, de 25 de fevereiro de 2003. Dispõe sobre o regulamento técnico para o gerenciamento de resíduos de saúde.Disponível em URL: http://www.anvisa.gov.br Acessado em: 12 abr 2003.

6. Ministério do Trabalho (BR). Associação Brasileira De Normas Técnicas Portaria $n^{0} 3.214$, de 08 de junho de 1978. Dispõe sobre Normas Regulamentadoras do Trabalho. Brasília (DF); 1978.

7. Ribeiro Filho VO. Gerenciamento de resíduos de serviços de saúde. In: Fernandes AT, Ribeiro Filho N, Fernandes MOV. Infecção hospitalar e suas interfaces na área de saúde. São Paulo:Atheneu;2001.

8. Associação Paulista de Controle de Infecção Hospitalar-APECIH. Orientações para o controle de infecções em pessoal da área de saúde. São Paulo:APECIH; 1998.

9. Bolyard EA, Tablan OC, Williams MW. Guideline for infection control in healthcare personnel. Infect Control Hosp Epidemiol 1998;19(6):41063

10. Ministério da Saúde (BR). Coordenação de DST/Aids. Manual de Condutas - exposição ocupacional a material biológico: HEAPTITE e HIV. Brasília (DF): MS;1998.

11. CDC. Update: U.S. Public health service guidelines for the management of occupational exposures to HBV, HCV and recommendations for post exposure prophylaxis. MMWR 2001 June 29;50(RR11):1-42.

12. Cavalcante NJF, Pereira NA. Saúde ocupacional. In: Fernandes AT, Fernandes MOV, Filho NR. Infecção hospitalar e suas interfaces na área da saúde São Paulo: Atheneu;2000.p.1287-300.

13. Secretaria de Estado da Saúde (GO). Coordenação Estadual de Controle de Infecção Hospitalar. Programa de prevenção e assistência ao acidente do profissional com material biológico: manual de orientação. Goiânia (GO): SES;2003. 24 p.

14. Garner JS. Guideline for isolation precautions in hospitals. Infect Control Hosp Epidemiol 1996;13(5):8-257.

15. Conceição FM, Cavalcante NJF, Ayub MA. Imunização em profissionais de saúde. In: Fernandes AT, Fernandes MOV, Filho NR. Infecção hospitalar e suas interfaces na área da saúde. São Paulo: Atheneu; 2000.p.1301-06.

16. Lopes CLR, Martins RMB, Teles AS, Silva AS, Maggl OS, Yoshida CFT. Perfil soroepidemiológico pelo vírus da Hepatite $\mathrm{B}$ em profissionais das unidades de hemodiálise de Goiânia - Goiás, Brasil Central. Revista da Sociedade de Medicina Tropical 2001 nov/dez; 34(6):54348

17. Lacerda RA. Risco de infecção ocupacional a substâncias orgânicas e medidas de precauções. In: Lacerda RA. Controle de infecção em centro cirúrgico. São Paulo Atheneu;2003.p.469-531.

Data de Recebimento: 22/06/2004

Data de Aprovação: 26/12/2004 